\title{
Estudo do perfil farmacológico de idosos hipertensos praticantes de atividades físicas do Programa Raízes da Vida
}

\author{
Jocicleide de Sousa Freitas", Francisco das Chagas Vasconcelos de Souza Neto", \\ Maira Elisa Grassi de Sá"**, Paula Matias Soares"**
}

\section{Resumo}

Este estudo busca verificar quais são os principais fármacos utilizados no tratamento da hipertensão arterial sistêmica (HAS) em idosos praticantes de exercícios físicos. Para tanto, a pesquisa apoiou-se em fichas preenchidas com os dados dos participantes (pesquisa documental). Constituiu-se do levantamento de 85 fichas de anamnese de idosos participantes do Programa Gerontológico Raízes da Vida do Instituto Federal de Educação, Ciência e Tecnologia do Ceará (IFCE). A análise foi realizada a partir de estatística descritiva, considerando frequência simples (dados brutos e percentuais) para análise dos dados. Como resultado, obteve-se que $62,3 \%$ dos idosos apresentavam HAS e faziam tratamento farmacológico. Praticamente todas as classes de anti-hipertensivos foram utilizadas no tratamento da HAS para esse grupo, com exceção dos vasodilatadores diretos. A proporção da utilização desses fármacos foi: antagonistas dos receptores de angiotensina (28\%), inibidores adrenérgicos (25\%), diuréticos (13\%), bloqueadores dos canais de cálcio (11\%), inibidores da enzima conversora de angiotensina $(4 \%)$, e combinações farmacológicas $(10 \%)$, apenas $18,9 \%$ do grupo utilizava combinação de classes, visto ser o mecanismo predominante a limitação do efeito vasoconstritor da angiotensina. Conclui-se que, para esse grupo, a medicação mais utilizada encontra-se dentro do grupo de antagonistas dos receptores de angiotensina e inibidores adrenérgicos, os quais, apesar de apresentarem mecanismos de ação diferentes, contribuem para a redução da pressão arterial.

Palavras-chave: Idoso. Hipertensão. Fármacos. Exercício físico.

* Graduanda em Educação Física pela Universidade Estadual do Ceará. E-mail: jocicleide11@gmail.com.

** Graduando em Educação Física pela Universidade Estadual do Ceará. E-mail: vasconcelos.18@gmail.com.

*** Especialista em Gerontologia. Mestre em Ciências da Educação pela Universidad Americana. Professora do Programa Gerontológico Raízes da Vida do Instituto Federal de Educação, Ciência e Tecnologia do Ceará (IFCE). E-mail: maira_grassi@hotmail.com.

***** Doutora em Biotecnologia (UFC/Renorbio). Universidade Estadual do Ceará. E-mail: dra.soares_pm@yahoo. com.br.

$\rightarrow$ http://dx.doi.org/10.5335/rbceh.2013.3156 


\section{Introdução}

No Brasil, a hipertensão arterial sistêmica (HAS) afeta mais de 30 milhões de pessoas (36\% dos homens adultos e $30 \%$ das mulheres) e representa o mais importante fator de risco para o desenvolvimento das doenças cardiovasculares, com destaque para o acidente vascular cerebral e o infarto do miocárdio, as duas maiores causas isoladas de mortes no país (SOCIEDADE BRASILEIRADE HIPERTENSÃO ARTERIAL, 2010).

A hipertensão arterial sistêmica é uma patologia de origem multifatorial, uma síndrome de evolução clínica lenta que se caracteriza por pressão arterial, a partir de 135/85 mmHg. Valores acima desse, favorecem o surgimento de alterações cardiovasculares e metabólicas, podendo acarretar alterações funcionais e estruturais em vários órgãos (SOCIEDADE BRASILEIRA DE HIPERTENSÃO, 2006). Assim, a identificação e o tratamento de pacientes com hipertensão arterial sistêmica constituem um problema de saúde pública no Brasil (MONTEIRO; SOBRAL FILHO, 2004).

Com o avanço da idade da população, a prevalência da HAS continuará a aumentar até que se adotem estratégias preventivas efetivas. Dados do Framingham Heart Study revelam que um indivíduo normotensivo, aos 55 anos, tem $90 \%$ de risco de desenvolver um quadro hipertensivo até o final da vida (KANNEL, 2004).

Parâmetros fisiopatológicos dessa doença estão, intimamente, relacionados ao processo de envelhecimento, haja vista que esse fenômeno promove alterações estruturais e funcionais no sistema vascular, mesmo na ausência de patologias. Isso deve-se, principalmente, aos valores da pressão arterial sistólica elevada e à redução da pressão arterial diastólica (MENDES; BARATA, 2008). Sabe-se que, ao longo dos anos, a resistência vascular sofre modificações decorrentes do processo de arteriosclerose, fenômeno natural do organismo humano (BERNE; LEVY, 2009). Além da idade, uma série de fatores, como gênero e etnia, excesso de peso e obesidade, ingestão de álcool e de sal, sedentarismo, e fatores socioeconômicos e ambientais são importantes desencadeadores no desenvolvimento da hipertensão (SOCIEDADE BRASILEIRA DE HIPERTENSÃO ARTERIAL, 2010).

A HAS pode ter também origem e influência genética. Assim, quanto mais parentes hipertensos um paciente tiver, maiores serão as possibilidades de desenvolver a patologia (NUNES; SILVA, 2013).

O tratamento da HAS dar-se-á por meio da adoção de medidas medicamentosas e não medicamentosas, as quais consistem na adoção de um estilo de vida saudável, que devem ser adotadas desde a infância. Sabe-se que a moderação na ingestão de sódio $(2,4 \mathrm{~g} /$ dia), álcool (30 $\mathrm{ml}$ de etanol/dia para homens e $15 \mathrm{ml}$ para mulheres) e gorduras saturadas; 0 consumo de alimentos ricos em potássio, magnésio, cálcio e fibras; a atividade física aeróbica regular, assim como perda de peso em obesos, são medidas importantes não somente na redução do uso de anti-hipertensivos em idosos, mas também na prevenção de outros fatores de riscos (MIRANDA et al., 2002). 
O tratamento dessa patologia tem por objetivo manter os valores pressóricos abaixo de 140/90 $\mathrm{mmHg}$. Dentro disso, o tratamento não medicamentoso é recomendado para todas as idades, principalmente para idosos. Entretanto, quando o tratamento medicamentoso é necessário, a dose inicial deve ser baixa e o incremento nas doses ou a associação de outros medicamentos devem ser feitos com cuidado (SOCIEDADE BRASILEIRA DE HIPERTENSÃO ARTERIAL, 2010).

Segundo as VI Diretrizes Brasileiras de Hipertensão (2010) os fármacos disponíveis, comercialmente, para o tratamento da patologia subdividem-se nas seguintes classes, com seus respectivos mecanismos de ação:

- diuréticos - efeitos diuréticos e natriuréticos, por meio da diminuição no volume extracelular. Após um mês, o volume circulante é, praticamente, normalizado e há redução da resistência vascular periférica;

- inibidores adrenérgicos - os inibidores adrenérgicos podem ser de três tipos: de ação central (reduzindo o tônus simpático por ação em receptores alfa-2-pré-sinápticos), betabloqueadores e alfabloqueadores (de ação cardiovascular);

- vasodilatadores diretos - atuam na musculatura da parede vascular, promovendo relaxamento muscular com consequente vasodilatação e redução da resistência vascular periférica;
- bloqueadores de canais de $\mathrm{Ca}^{+2}-$ promove a redução da resistência vascular periférica através da diminuição da concentração de cálcio nas células musculares lisas do tecido vascular;

- inibidores da ECA - atuam inibindo a enzima conversora da angiotensina (ECA), bloqueando a transformação da angiotensina I em II no sangue e nos tecidos (atenuação do efeito vasoconstrictor);

- bloqueadores dos receptores AT1 da angiotensina II - antagonizam a ação da angiotensina II por meio do bloqueio específico de seus receptores AT1;

- inibidores diretos da renina - o Alisquireno é o único representante da classe, atualmente, disponível para uso clínico. Esse promove inibição direta da ação da renina com consequente diminuição da formação de angiotensina II. Especulam-se, ainda, outras ações, como redução da atividade plasmática de renina, bloqueio de um receptor celular próprio de renina/prorrenina e diminuição da síntese intracelular de angiotensina II.

Essa variedade no tratamento gera um problema frequente na aderência ao tratamento anti-hipertensivo, não apenas na população jovem, como também na idosa. Esse, provavelmente, é o maior desafio enfrentado hoje para o controle adequado, em larga escala, da hipertensão (MIRANDA et al., 2002). Além disso, para prescrever um treinamento adequado para esses indivíduos, é necessário 
conhecimento prévio acerca da ingestão de medicamentos e o tipo específico desse no intuito de adequar todos os parâmetros relacionados ao treinamento, como tipo de exercício, intensidade e volume da atividade.

Em vista disso, a presente pesquisa busca verificar, quais as principais classes de fármacos utilizados no tratamento dessa patologia, em um grupo praticante de exercício físico.

\section{Método}

Este estudo utilizou-se da pesquisa documental para levantamento dos dados, a qual realizou-se a partir da análise de 85 fichas de anamnese de idosos praticantes de exercícios físicos (ginástica, musculação, hidroginástica e dança sênior) do Programa Gerontológico Raízes da Vida do Instituto Federal de Educação, Ciência e Tecnologia do
Ceará. Para análise dos resultados, foram utilizadas estatística descritiva por meio de frequência simples (dados brutos e percentuais).

Ressalta-se ainda que o projeto de pesquisa deste estudo foi avaliado e aprovado pela Comissão de Ética em Pesquisa da Universidade de Fortaleza (CEP/Unifor), com protocolo n. 206/2008.

\section{Resultados e discussão}

A partir das 85 fichas avaliadas de indivíduos acima de 60 anos e praticantes de exercício físico, observou-se que $62,3 \%$ do grupo estudado apresentavam HAS. Salienta-se que todos os idosos que afirmaram ter HAS diagnosticada também fizeram menção ao fato de utilizar algum tipo de medicamento. A Figura 1 mostra a distribuição percentual das classes de anti-hipertensivos, citados pelos idosos, em suas fichas.

Figura 1 - Distribuição percentual de fármacos anti-hipertensivos utilizados pelo grupo em estudo

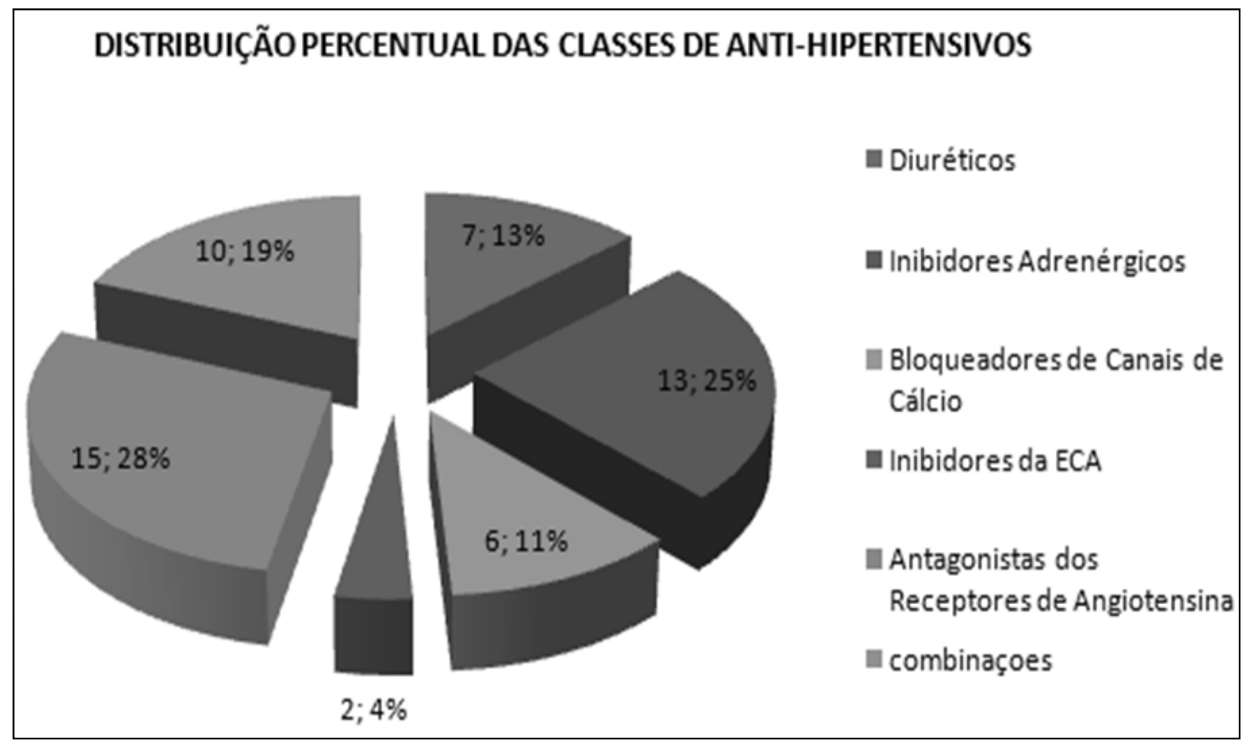


Com base nesse levantamento, pode-se afirmar que as classes de anti-hipertensivos mais prevalentes são: antagonistas de receptores de angiotensina (28\%) e inibidores adrenérgicos (25\%). Enquanto que a classe que aparece em menor proporção é a dos inibidores da Enzima Conversora de Angiotensina (ECA) com um valor percentual de $4 \%$.

Um estudo realizado com idosos não praticantes de exercícios físicos, integrantes de uma unidade terapêutica localizada em São Paulo, mostrou resul- tados contrastantes com aqueles obtidos nesta pesquisa, em que a classe de medicamentos mais utilizada foi a dos inibidores da ECA (LONGO; MARTELLI; ZIMMERMANN, 2011). Observa-se que, apesar da classe ser diferente, o resultado final é semelhante, pois ambos interferem na ação vasoconstritora.

No Quadro 1 encontram-se as combinações de fármacos utilizados pelos idosos participantes (18,9\% do grupo que apresentava HAS) com seus respectivos nomes comerciais e princípios ativos.

Quadro 1 - Distribuição dos dados brutos e percentual dos indivíduos que utilizavam combinação farmacológica para tratamento da hipertensão

\begin{tabular}{|c|c|c|}
\hline Nome comercial & $\begin{array}{l}\text { Quantidade de } \\
\text { usuários (n e \%) }\end{array}$ & Componentes \\
\hline Micards & $4(40 \%)$ & $\begin{array}{l}\text { Antagonista do receptor de angiotensina II e diuréti- } \\
\text { co tiadízico (hidrocloritiazida). }\end{array}$ \\
\hline Bennicar & $1(10 \%)$ & $\begin{array}{l}\text { Bloqueadores de canais de } \mathrm{Ca}^{+2} \text { e bloqueador de } \\
\text { receptores de angiotensina II. }\end{array}$ \\
\hline Diovan amlo fix & $4(40 \%)$ & $\begin{array}{l}\text { Antagonista dos receptores de angiotensina II e } \\
\text { bloqueadores de } \mathrm{CCa}^{+2} \text { derivados de diidropiridina. }\end{array}$ \\
\hline Aradois $\mathrm{H} 100$ & $1(10 \%)$ & $\begin{array}{l}\text { Antagonista do receptor de angiotensina II (losarta- } \\
\text { na) e diurético tiazídico (hidrocloritiazida). }\end{array}$ \\
\hline
\end{tabular}

Nos dados de Longo, Martelli e Zimmermann (2001), 60\% dos pacientes com HAS necessita de terapia combinada, enquanto que, neste estudo apenas 18,9\% utilizavam combinação de fármacos. Isso, provavelmente, pode ter sido diferente pelo fato de a nossa amostra ser ativa e a do outro estudo sedentária. Miranda et al. (2002) afirmam que o uso da terapia farmacológica combinada (duas drogas no mesmo comprimido) é uma necessidade para os idosos, pois atua melhorando a aderência e a eficácia anti-hipertensiva e diminuindo os efeitos colaterais das substâncias associadas.

A utilização de fármacos anti-hipertensivos, destinados ao paciente idoso, deve considerar algumas particularidades desse grupo. É preciso atentar-se para as alterações próprias do envelhecimento, a presença de outras patologias, $o$ número de doses diárias do medicamento, possíveis interações medicamentosas e o estilo de vida relacionado à alimentação e à prática regular de exercícios físicos (RAMOS et al., 1998). 
Em relação às alterações fisiológicas que ocorrem no indivíduo idoso, tem-se a alteração da função cardiovascular, que pode resultar em aumento da pressão arterial. Essa alteração no sistema cardiovascular ocorre, principalmente, em decorrência do processo de arteriosclerose, aumentando, assim, a resistência vascular periférica (BERNE; LEVY, 2009).

A prática de exercícios físicos, programada e orientada, acarreta diversos benefícios ao indivíduo idoso e em relação ao sistema cardiovascular não é diferente. À medida que o indivíduo envelhece, sua resistência cardiovascular diminui e a extensão dessa diminuição depende do nível de atividade física e do patrimônio genético do idoso. Sabe-se que com o declínio do nível de atividade, essas reduções na função biológica tornam-se mais expressivas (COSTILLI; KENNEY; WILMORE, 2010).

Segundo Nogueira et al. (2012), o efeito protetor do exercício físico vai além da redução pressórica, haja vista que o exercício físico regular está associado à redução de fatores de risco cardiovasculares e à menor morbimortalidade.

Em situações de exercício, diversas modificações fisiológicas são realizadas pelo organismo, a fim de atender às crescentes demandas necessárias para sua realização. Costilli, Wilmore e Kenney (2010) citam os seguintes fatores fisiológicos que são modificados em uma situação de exercício e que afetam o sistema cardiovascular:

- aumento da frequência cardíaca de repouso (resposta antecipatória ao exercício); do volume sistólico, do débito cardíaco e da pressão arterial (o aumento da pressão arterial é uma resposta ao aumento do débito cardíaco). Salienta-se que a pressão arterial sistólica é a que mais aumenta, enquanto a pressão arterial diastólica quase não se altera;

- redistribuição do fluxo sanguíneo para regiões de maior necessidade - por meio do sistema nervoso simpático o fluxo sanguíneo é redirecionado para as áreas que são ativas durante a atividade. Esse redirecionamento é possível devido, principalmente, à redução desse fluxo para os rins e para a circulação esplênica (fígado, estômago e intestinos);

- redução do volume sanguíneo - o sangue tem papel importante durante o exercício físico, visto que ele é responsável pelo transporte de substâncias necessárias para os tecidos, para o recolhimento e para a eliminação de produtos inúteis do metabolismo. Com o início do exercício, ocorre uma perda praticamente imediata de plasma sanguíneo para o espaço que contém o líquido intersticial. Esse volume pode reduzir consideravelmente se as condições ambientais induzirem à produção de suor. A redução do plasma sanguíneo diminui, ocorrendo a hemoconcentração, quer dizer, a parte líquida do sangue fica reduzida, e as partes celular e proteica representam maior fração do volume sanguíneo total. 
Todas essas modificações devem ser levadas em consideração quando se prescreve treinamento físico para o indivíduo hipertenso, sobretudo ao tratar-se de um idoso sob a utilização de medicamento. Assim, a relação entre o fármaco utilizado pelo idoso para tratar a hipertensão, deve ser considerada partindo-se do mecanismo de atuação desse medicamento, com o indivíduo em repouso e a influência desse nos fatores que se modificam com o exercício.

Viu-se na presente pesquisa que a classe de anti-hipertensivo mais utilizado pelos idosos foi a do antagonistas de receptores AT1 de angiotensina II (28\%). Sabe-se que a ação desse consiste no bloqueio de canais do tipo AT1, impossibilitando a formação de angiotensina II, além de se constituir na classe de anti-hipertensivos que apresentam menor risco de efeitos colaterais. É sabido também que a angiotensina II tem um importante papel vasoconstrictor, que não é cumprido na ausência dessa. Sabe-se que os antagonistas dos receptores da angiotensina II têm como mecanismo de ação o bloqueio das ações dessa pela ocupação específica do receptor AT1 exercendo, consequentemente, ação anti-hipertensiva e protetora para os diferentes órgãos-alvo da hipertensão arterial (RANG et al., 2011).

A angiotensina II apresenta diversos efeitos no organismo e atua, principalmente, via receptor AT-1. A angiotensina II age nos centros hipotalâmicos, gerando aumento da sede e do apetite por sal, na musculatura lisa vascular criando vasoconstrição sistêmica, na neurohipófise elevando a liberação de hormônio antidiurético, além de estimu- lar a proliferação das células musculares lisas. Desse modo, a angiotensina II aumenta o volume circulante efetivo e gera potente vasoconstrição (BORON; BOULPAEP, 2003).

Partindo-se do pressuposto de que, ao ingerir um fármaco anti-hipertensivo pertencente à classe antagonista de receptores AT1 de angiotensina II, o paciente está impedindo a formação do peptídeo e, portanto, interferindo no processo natural que aconteceria com o indivíduo em exercício. Como discutido anteriormente, ao iniciar um exercício físico, o volume plasmático diminui consideravelmente. Essa queda do volume circulante produz uma queda de pressão que estimula a liberação de renina. A renina é uma enzima que converte o angiotensinogênio em angiotensina I. Após a formação da angiotensina I, essa entra na corrente sanguínea e é convertida em angiotensina II por meio da enzima conversora de angiotensina (ECA). A angiotensina II, depois de formada, pode atuar em dois tipos de receptores: o receptor AT1 (responsável pelos efeitos conhecidos da angiotensina II) e o receptor AT2. Quando o sujeito ingeriu o medicamento, a angiotensina II não pode se ligar ao receptor AT1, logo não pode exercer seus efeitos de vasoconstricção vascular; liberação de hormônio antidiurético e, portanto manutenção/aumento do volume plasmático; liberação de aldosterona com consequente estimulação da reabsorção de sódio.

Assim, pode-se dar algumas orientações em relação à prática de exercícios para idosos hipertensos que fazem uso de anti-hipertensivos da classe dos antagonistas dos receptores de angiotensina II. 
A primeira recomendação é a ingestão hídrica, durante a atividade, uma vez que o medicamento impede a liberação do $\mathrm{ADH}$ e, portanto, o indivíduo fica sujeito a uma queda acentuada do volume plasmático.

É importante salientar que o tratamento do indivíduo hipertenso deve ser um procedimento multiprofissional, ou seja, cada caso deve ser discutido pela equipe que acompanha o idoso. Caso não seja possível estabelecer uma discussão com toda a equipe que acompanha o indivíduo, que esta seja feita ao menos com o profissional de Educação Física e o médico responsável pelo paciente. $\mathrm{O}$ diálogo estabelecido pelos profissionais possibilitará um aproveitamento maior da terapia medicamentosa e da não medicamentosa, pois questões como a dosagem do fármaco e o tipo de medicamento e suas associações são questões discutíveis quando se pensa no idoso hipertenso fisicamente ativo.

Laterza et al. (2008) declaram que o treinamento físico aeróbio pode promover a redução pressórica mesmo naqueles pacientes hipertensos que estão sob a utilização de medicamentos anti-hipertensivos. A adoção dessa medida não medicamentosa pode acarretar diminuição da dose ou, em alguns casos, até a suspensão da medicação anti-hipertensiva. Entretanto, os autores afirmam que o efeito anti-hipertensivo do treinamento físico desaparece, após a interrupção do programa de treinamento e os mecanismos que modulam a redução pressórica (após um período de treinamento físico) ainda não estão totalmente elucidados.

A angiotensina II parece participar da regulação central da atividade nervosa simpática. E seus níveis centrais encontram-se aumentados em indivíduos com hipertensão arterial, o que, por sua vez, contribui diretamente para os altos níveis de atividade nervosa simpática observada nessa doença (LATERZA et al., 2008). Por outro lado, Felix e Michelini (2007) demonstraram que três meses de treinamento físico foram capazes de normalizar os níveis do RNA mensageiro do angiotensinogênio em ratos espontaneamente hipertensos. Tais resultados sugerem que a menor ativação central do sistema renina-angiotensina pode estar envolvida na diminuição da atividade nervosa simpática, observada na hipertensão arterial, após o treinamento físico. Isso pode ser considerada outra evidencia de que a administração do anti-hipertensivo tem de ser controlada conforme a progressão do programa de treinamento físico e o tratamento da hipertensão arterial.

A segunda recomendação consiste na ingestão dos antagonistas dos receptores de angiotensina II antes da prática do exercício físico, pelo menos no início do programa de exercícios. Visto que no pré-exercício físico ocorre o aumento da pressão arterial e da frequência cardíaca; e que no pós-exercício, em relação aos níveis pré-exercício, ocorre a redução da pressão arterial, essa pronunciada nos indivíduos hipertensos em comparação com os normotensos (HALLIWILL JR., 2001; MACDONALD JR., 2002). Portanto, essa recomendação visa à segurança do aluno/ cliente, uma vez que esse já está sob uma condição em que a sua pressão arterial já é aumentada em níveis basais, logo, em situação de exercício, pode aumentar de forma substancial e levar o indivíduo a alguma situação de desconforto. 
Além disso, com o prosseguimento do treinamento físico regular, pode-se discutir com o médico a mudança no horário de ingestão do fármaco, uma vez que os benefícios supracitados do exercício físico em relação à pressão arterial tornam-se mais evidentes.

\section{Conclusão}

A partir dos resultados, pode-se concluir que os medicamentos mais utilizados por idosos hipertensos ativos são antagonistas dos receptores de angiotensina e inibidores adrenérgicos, os quais apresentam particularidades que devem ser consideradas quanto à prescrição do treinamento físico. Percebeu-se, também, que uma parte menor do grupo utiliza associação de medicamentos, o que pode estar relacionada à prática constante de exercício físico, em virtude de não condizer com a maior parte dos artigos encontrados que afirmam ser mais comum a polimedicação, no caso de indivíduo com HAS.

O exercício físico regular, combinado à terapia medicamentosa, pode trazer diversos benefícios a esse público, como redução na dose de medicamentos, adoção da monoterapia e até a retirada do fármaco do tratamento da HAS. Entretanto, estudos mostram que o efeito anti-hipertensivo do treinamento físico desaparece após a interrupção do programa de treinamento e os mecanismos que modulam a redução pressórica (após um período de treinamento físico), ainda não estão totalmente elucidados.
Study of the pharmacological profile of hypertensive elderly physical activities practitioners of the Roots of Life Program

\section{Abstract}

This study sought to verify which are the main drugs used in the treatment of hypertension $(\mathrm{SAH})$ in elderly practitioners of physical exercises. To this end, the research supported in chips filled with the data of the participants (desk research). Consisted of a survey of 85 elderly participants history sheets of the Geriatric Program Roots of the life of the Federal Institute of education, science and technology of Ceará. The analysis was carried out from descriptive statistics, considering simple frequency (raw data and percentages) for data analysis. As a result it was obtained that the elderly had 62.3 HAS and pharmacological treatment of same. Practically all classes of antihypertensive drugs used in the treatment of HAS for this group, with the exception of direct vasodilators. The proportion of the use of these drugs was: angiotensin receptor antagonists (28), adrenergic inhibitors (25), diuretics (13), calcium channel blockers (11), angiotensin-converting enzyme inhibitors (4), and pharmacological combinations (10), where only 18.9 of the group used combination of classes, being the predominant mechanism limiting the vasoconstrictor effect of angiotensin. As a result it was obtained that the elderly had 62.3 HAS and pharmacological treatment of same. Practically all classes of antihypertensive drugs used in the treatment of HAS for this group, with the exception of direct vasodilators. The proportion of the use of these drugs was: angiotensin receptor antagonists (28), adrenergic inhibitors (25), diuretics (13), calcium channel blockers (11), angiotensin-converting enzyme inhibitors (4), and pharmacological combinations (10), where only 18.9 of the group used combination of classes, being the predominant mechanism limiting the vasocons- 
trictor effect of angiotensin. It is concluded that, for this group, the most widely used medication lies within the Group of angiotensin receptor antagonists and inhibitors, adrenergic receptors, which, despite performing different action mechanisms, contribute to the lowering of blood pressure.

Keywords: Elderly. Hypertension. Pharmaceuticals. Physical exercise.

\section{Referências}

BARROSO, W. K. S. et al. Influência da atividade física programada na pressão arterial de idosos hipertensos sob tratamento não-farmacológico. Revista da Associação Médica Brasileira, [online], São Paulo, v. 54, n. 4 , p. 328-333, jul./ago. 2008.

BERNE, R. M.; LEVY, M. N. Fisiologia. 6. ed. Rio de Janeiro: Editora Elsevier, 2009.

BORON, W. F.; BOULPAEP, E. L. Medical physiology. Philadelphia, PA: Elsevier Saunders, 2003.

COSTILLI, D. L.; KENNEY, W. L.; WILMORE, J. H. Fisiologia do esporte e do exercício. Barueri:, Manole, 2010.

FELIX, J. V. C.; MICHELINI, L. C. Training-induced pressure fall in spontaneously hypertensive rats is associated with reduced angiotensinogen mRNA expression within the nucleus tractus solitarii. Hypertension, São Paulo, v. 50, n. 4, p.780-85, jul. 2007.

HALLIWILL, Jr. Mechanisms and clinical implications of post-exercise hypotension in humans. Exercise Sport Science Review, Minnesota, v. 29, n. 2, p.65-70, Apr. 2001. Disponível em: <http://www.ncbi.nlm.nih. gov/pubmed/11337825>. Acesso em: 20 jan. 2013.

ISHIKAWA, K. et al. Influence of age and gender on exercise training-induced blood pressure reduction in systemic hyperten- sion. The American Journal of Cardiology, Tokyo, v. 84, n. 2, p. 192-196, July. 1999. Disponível em: < http://www.ncbi.nlm.nih. gov/pubmed/?term=Influence+of+age+and +gender+on+exercise+training-induced $+b$ lood+pressure+reduction+in+systemic+hy pertension>. Acesso em: 2 fev. 2013.

KANNEL, W. B. Hypertension as a risk factor: the framingham contribuition. In: BIRKENHAGER, W. H.; ROBERTSON, J. I. S.; ZANCHETTI. A. (Eds.). Handbook of hypertension. Elsevier, 2004. p. 129-142.

LATERZA, M. C. et al. Exercício físico regular e controle autonômico na hipertensão arterial. Revista Sociedade de Cardiologia do Estado do Rio de Janeiro, Rio de Janeiro, v. 21 , n. 5 , p. 320-328, set./out. 2008. Disponível em:<http://www.ufjf.br/caminhada/ files/2009/02/socerj-laterza-mc1.pdf $>$. Acesso em: 3 fev. 2013.

LONGO, M. A. T.; MARTELLI, A.; ZIMMERMANN, A. Hipertensão arterial sistêmica: aspectos clínicos e análise farmacológica no tratamento dos pacientes de um setor de Psicogeriatria do Instituto Bairral de Psiquiatria, no município de Itapira, SP. Revista Brasileira de Geriatria e Gerontologia, Rio de Janeiro, v. 14, n. 2, p. 271-328, dez. 2011.

MACDONALD, J. R. Potential causes, mechanisms, and implications of post exercise hypotension. Journal of Human Hypertension, Ontário, v. 16, n. 4, p. 225-236, Apr. 2002.

MENDES, R.; BARATA, J. L. T. Envelhecimento e pressão Arterial. Acta Médica Portuguesa, local de publicação, v. 21, n. 2, p. 193-198, jan. 2008. Disponível em: <http:// www.actamedicaportuguesa.com/revista/ index.php/amp/article/view/769/446>. Acesso em: 20 maio 2014 .

MIRANDA, R. D. et al. Hipertensão arterial no idoso: peculiaridades na fisiopatologia, no diagnóstico e no tratamento. Revista Brasileira de Hipertensão, Ribeirão Preto, v. 9, n. 3, p. 293-300, jun. 2002.

MONTEIRO, M. F.; SOBRAL FILHO, D. C. Exercício físico e o controle da pressão 
arterial. Revista Brasileira de Medicina do Esporte, São Paulo, v. 10, n. 6, p. 513-516, nov./dez. 2004.

NOGUEIRA, I. C. et al. Efeitos do exercício físico no controle da hipertensão arterial em idosos: uma revisão sistemática. Revista Brasileira de Geriatria e Gerontologia, Rio de Janeiro, v. 15, n. 3, p. 587-601, jul./set. 2012. Disponível em: <http://www.scielo.br/scielo.php?pid=S1809$-98232012000300019 \&$ script=sci_arttext $>$. Acesso em: 20 mar. 2013.

NUNES, A. L. S. C.; SILVA, C. B. Adesão da educação para o tratamento da hipertensão arterial crônica. 2013. Trabalho de conclusão de curso (Graduação em Enfermagem) - Faculdades Integradas Promove de Brasília, Brasília, 2013. Disponível em: <http://nippromove.hospedagemdesites.ws/anais_simposio/ arquivos_up/documentos/artigos/705817191 0c0cffb617f9f09d640bbae.pdf $>$. Acesso em: 24 maio 2014.

RAMOS, L. R. et al. Two-year follow-up study of elderly residents in São Paulo, Brazil (EPIDOSO Project): Methodology and preliminary results. Revista de Saúde Pública, São Paulo, v. 32, n. 5, p. 397-407, Oct. 1998. Disponível em: <http://www.scielo. br/scielo.php?script=sci_arttext\&pid=S0034$-89101998000500001 \&$ lng $=\mathrm{en}>$. Acesso em: 24 maio 2014.

RANG, H. P. et al. Farmacologia. 7. ed. Rio de Janeiro: Ed. Elsevier, 2011.

SOCIEDADE BRASILEIRADE HIPERTENSÃO. Diretrizes Brasileiras de Hipertensão VI. Revista Brasileira de Hipertensão, Ribeirão Preto, v. 13, n. 01, p. 1-64, jan./mar. 2010. Disponível em: <http://www.saude.al.gov.br/ sites/default/files/VI_Diretrizes_Bras_Hipertens_RDHA.pdf>. Acesso em: 25 mar. 2013. 